\title{
Architecture of the dorsal and ventral lobes of the prostate of the Syrian hamster, Mesocricetus auratus, after regrowth from short day-induced regression
}

\author{
G. R. Buzzell \\ Department of Anatomy and Cell Biology, University of Alberta, Edmonton, Alberta, \\ Canada $T 6 G 2 H 7$
}

\begin{abstract}
Summary. The structures of the dorsal and ventral lobes of the prostate of 4 groups of Syrian hamsters were studied by a stereological approach. Groups studied were young hamsters (kept in long-day photoperiods for 4 weeks) in their first breeding season, older hamsters (kept in short-day photoperiods for 24 weeks so that their prostates had regressed and regrown) in their second breeding season, and two groups of older hamsters in an extended first breeding season (either because they were kept in long days for 24 weeks or because they were pinealectomized before being put into shortday photoperiods for 24 weeks). There were very few differences between groups, but generally dorsal prostates of older hamsters in their second breeding season closely resembled those of young hamsters in their first breeding season. More differences were noted between either of these two groups and older hamsters in an extended first breeding season than between these two groups. The differences noted generally involved increases in the amount of smooth muscle in the walls of secretory tubules in the dorsal prostates of hamsters in an extended first breeding season. This may be associated with the fact that these glands had not regressed and regrown. Ventral prostates were very similar in all 4 groups, which may reflect the fact that they normally regress very little in short days.
\end{abstract}

Keywords: dorsal prostate; ventral prostate; pineal; stereology; seasonal reproduction; hamsters

\section{Introduction}

Syrian hamsters are seasonal breeders. In their normal environment, they reproduce during the long days of the spring and summer. As the days shorten near the autumnal equinox, the animals enter a period of reproductive quiescence; reproductive hormone concentrations drop and sexual organs regress. This reproductive regression in response to short days depends upon the presence of an intact and innervated pineal gland (Reiter \& Hester, 1966). After about 8-10 weeks in short days, this gondal regression is complete and the gonads remain regressed for a further 8-10 weeks. Following this period of regression, the endocrine and reproductive systems become refractory to pineal and short photoperiod influences; prolactin and gonadotrophin concentrations rise and the gonads regrow (Turek et al., 1975; Matt \& Stetson, 1980; Goldman et al., 1981; Steger et al., 1982; Reiter, 1985).

In male hamsters, the dorsal lobes of the prostate reflect this seasonal pattern. In long days, they are large and contain abundant secretion. In short days, they regress over a period of about 10 weeks (Buzzell et al., 1985b) and regrow to their original size by Week 24 in short days (this study). The ventral lobes of the prostate, on the other hand, are relatively resistant to short days; their regression in these conditions is not very marked (Buzzell et al., 1985a, b). The purpose of this study 
was to determine whether the seasonal regrowth of the prostate restores this gland to its original condition.

\section{Materials and Methods}

Animals and experimental procedures. Male Syrian hamsters, Mesocricetus auratus, were obtained from Charles River Laboratories, Montreal, at 8 weeks of age. They were housed 3 or 4 to a cage, in controlled conditions of lighting and temperature $\left(22^{\circ} \mathrm{C}\right)$, and provided with food and water ad libitum. The hamsters in Group $4(\mathrm{~N}=7)$ were pinealectomized by the method of Hoffman \& Reiter (1965). The pinealectomized hamsters and intact hamsters in Group 3 $(\mathrm{N}=10)$ were placed in a short-day photoperiod (10 h light:14 h dark, lights on 08:00-18:00 h). The intact hamsters in Groups 1 and $2(\mathrm{~N}=10$ in each) were maintained in a long-day photoperiod (14L:10D, lights on 06:00-20:00 h).

Hamsters in Group 1 were sampled after 4 weeks and those of the other 3 groups were sampled after 24 weeks. Hamsters were decapitated; the dorsal and ventral lobes of the prostate were dissected, weighed, and fixed in neutral buffered formalin. After fixation, each dorsal lobe was divided into two pieces, which were embedded in glycol methacrylate (JB-4, Polysciences, Inc., Warrington, PA, USA). Ventral lobes were embedded whole. Sections $(2 \mu \mathrm{m})$ were cut at random orientations from these blocks and stained with methylene blue-basic fuchsin.

Stereology. Prostatic architecture was assessed stereologically. The basic approach has been described (Buzzell, 1985), but I have modified the published techniques as outlined below.

The sections (one from each block) were projected at $\times 53$ magnification onto a piece of paper. Outlines of the section and of all secretory tubules were drawn. In the dorsal lobe, each tubule was graded as being 'folded' or 'distended', depending on the state of the lumen and mucosa. This was done because of reports that these regions are physiologically significant in some rodents (Sugimura et al., 1986a, b). Distended tubules were judged to have no mucosal folds which projected more than halfway to the centre of the tubule. This degree of folding and distension was not apparent in the ventral lobe (Toma \& Buzzell, 1988), and so, in this gland, all tubules were treated alike. The tracings were then measured on a digitizing pad. The area of the section was measured, followed by the areas of all tubules in the ventral lobes and of folded and of distended tubules in the dorsal lobe. From these data, the volume fractions of tubules and interacinar tissue, and, in the dorsal prostate, the volume fractions of folded and distended tubules were calculated from their area densities (Weibel, 1979). In addition, the number of each type of tubular profile was counted and the length fractions of tubules were calculated from the number of profiles and the area of the section (Buzzell, 1985). All of these fractions were calculated with reference to the volume of the gland.

The sections were then examined microscopically. Sections to be measured were selected at random. From the dorsal lobes, 10, 17,15 and 11 sections were measured in Groups 1,2,3 and 4, respectively. From the ventral lobes, 16, 16,14 and 17 sections were measured in Groups 1,2,3 and 4, respectively. Counts were made with a stereology graticule of the numbers of points and intercepts falling on areas of interest within the field of view. In each field of view, points falling in the tubular lumen, epithelium, lamina propria, and muscular stroma were counted; around 1000 points both on folded and distended tubules were counted in each section of the dorsal prostate; a total of 1500-2000 points was counted in each section of the ventral prostate. For each field of view sampled, intercepts of test lines between lumen and epithelium, epithelium and lamina propria, lamina propria and muscular stroma, and muscular stroma and interacinar tissue were counted. All counts were done at a magnification of $\times 250$; the stereology graticule had 100 points $(10 \times 10)$ and $3.733 \mathrm{~mm}$ of test lines, at this magnification.

Volume fractions of lumen, epithelium, lamina propria, and muscular stroma, with reference to the tubular volume, were calculated from the points counted. The intercept counts were used to calculate surface fractions of inner and outer borders of the epithelium, lamina propria, and muscular stroma (Weibel, 1979; Buzzell, 1985).

The absolute volume of the prostate was calculated from its weight and specific gravity (1.064) (Buzzell, 1985). From this volume and the volume, surface and length fractions, absolute values of the volumes and lengths of tubules, volumes of interacinar tissues, and volumes and surface areas of each component of tubules, were calculated.

Typical tubular characteristics calculated included the tubular radius. This was calculated using the formula for the radius of a cylinder from its volume and its length, on the assumption that acini are tubular in shape. The thicknesses of epithelium, lamina propria, and muscular stroma were then calculated from the formula for the height of a prism whose surface areas (top and bottom) and volume are known. Finally, the degree of tubular folding was assessed using a folding factor, as previously described (Buzzell, 1985).

Data management. Data are expressed as mean \pm standard error of the mean (s.e.m.). One-way analysis of variance (ANOVA) was performed on the data. Differences between groups were considered to be significant at $P<0 \cdot 05$. When significant differences were indicated by the ANOVA, the source of these differences was determined by the Student-Neuman-Keul's test.

\section{Results}

Detailed tables giving the results of measurements on the dorsal and ventral lobes of the prostate in these experimental conditions are available upon request to the author. The comparisons between the groups in the dorsal lobes are summarized in Figs 1 and 2. 


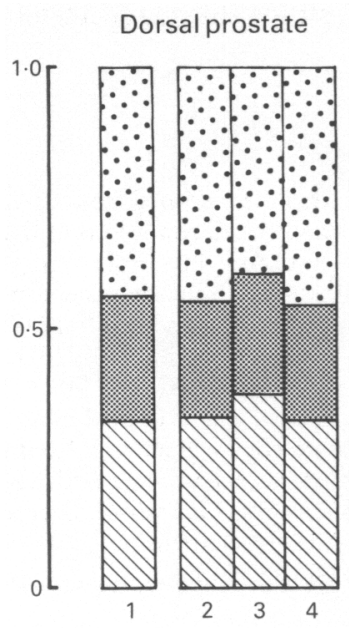

Distended acini
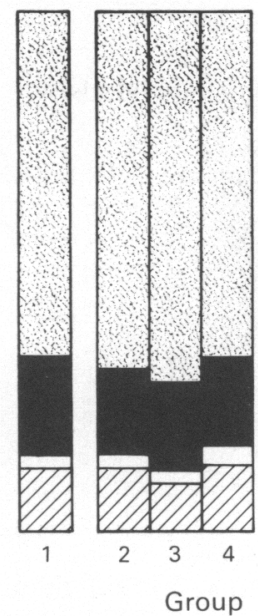

Folded acini
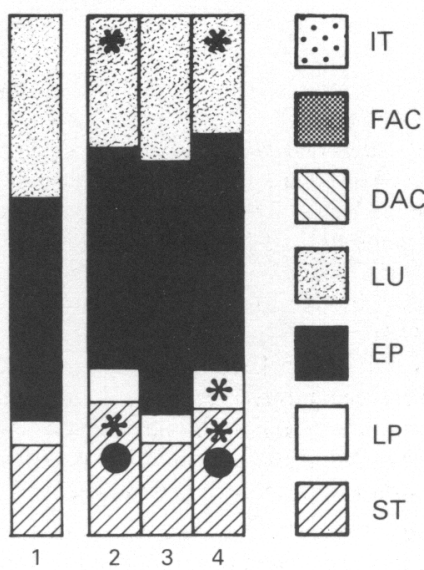

Fig. 1. Volume fractions of components of the dorsal prostate and of distended and folded acini within the dorsal prostate. Volume fractions within the dorsal prostate represent the proportion of the volume of the gland occupied by each component. Within the distended or folded acini, the volume fractions represent the proportion of the volume of the acinus occupied by each component. Group 1, intact hamsters kept in long days for 4 weeks; Group 2, intact hamsters kept in long days for 24 weeks; Group 3, intact hamsters kept in short days for 24 weeks; Group 4, pinealectomized hamsters kept in short days for 24 weeks. DAC, distended acini; EP, epithelium; FAC, folded acini; IT, interacinar tissue; LP, lamina propria; LU, lumen; ST, muscular stroma. ${ }^{*} P<0.05$ compared with Group 1; $P<0.05$ compared with Group 3.

The mean weights and hence the volumes, of the dorsal prostates did not differ in the 4 experimental groups. The volume fractions of the gland which were composed of tubules and of interacinar tissue also did not differ between groups (Fig. 1). In each case, the tubular compartment of the gland consisted of about $60 \%$ distended tubules and $40 \%$ folded tubules.

Within the dorsal prostate, neither tubular length fractions nor total lengths of distended tubules differed in the four groups except that length fraction in distended tubules was significantly greater in Group 4 than in Group 3 animals. In folded tubules, no significant differences were noted in tubular length fractions or total tubular length. The total volume of secretion within the glands did not differ significantly between the 4 groups.

Within distended tubules of the dorsal prostate, the proportions of the tubules composed of lumen, epithelium, lamina propria, and muscular stroma were similar in the 4 groups (Fig. 1). The surface areas of the tubules and their different components did not differ in distended tubules.

Typical distended tubules of the dorsal prostate were wider in Group 3 animals than in those of the other groups and this difference was significant when compared with that in Group 4 hamsters (Fig. 2). The lamina propria was significantly thicker in Group 4 than in Group 1 hamsters. The thicknesses of none of the other layers of the wall of distended tubules differed between groups. The folding factors also did not differ between groups in distended tubules.

Within folded tubules of the dorsal prostate, hamsters in Groups 2 and 4 had smaller volume fractions of lumen than did hamsters in Group 1 and greater volume fractions of muscular stroma than did those in Groups 1 and 3 (Fig. 1). The surface areas of the tubules and their different components did not differ between the groups of folded tubules.

Radii of folded tubules of the dorsal prostate did not differ in the 4 groups. However, the thickness of the muscular stroma in Group 1 animals was less than that of Groups 2 and 4 and 


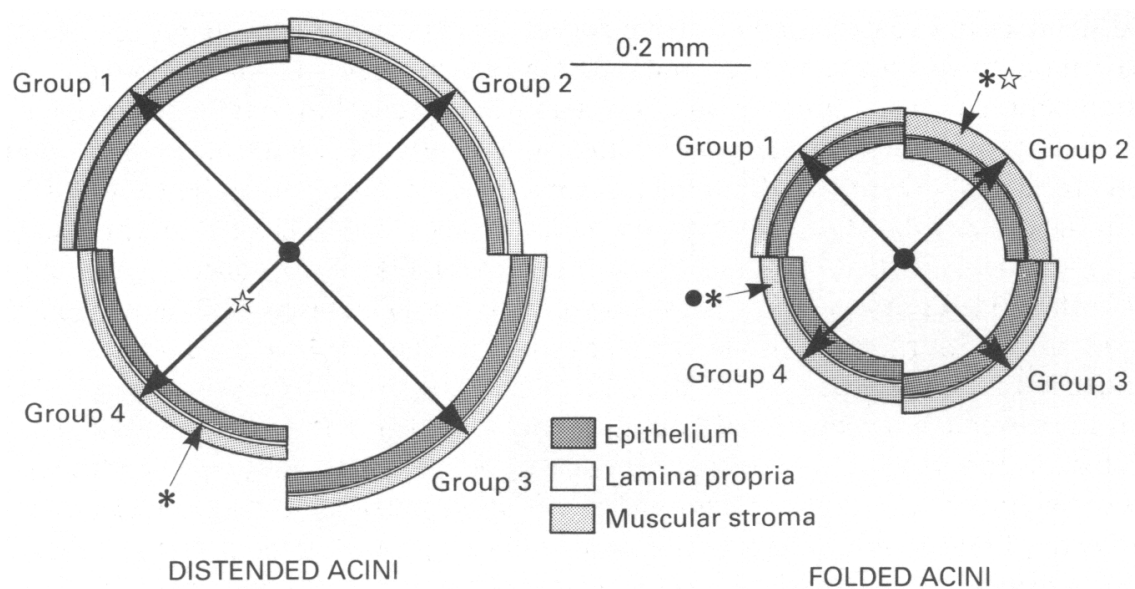

Fig. 2. Wedges $\left(90^{\circ}\right)$, representing typical radii and thicknesses of acinar components in distended and folded acini from dorsal prostates of hamsters subjected to the experimental procedures. Group 1, intact hamsters kept in long days for 4 weeks; Group 2, intact hamsters kept in long days for 24 weeks; Group 3, intact hamsters kept in short days for 24 weeks; Group 4, pinealectomized hamsters kept in short days for 24 weeks. ${ }^{*} P<0.05$ compared with Group 1; $P<0.05$ compared with Group 3; $P<0.05$ compared with Group 2.

similar to that of Group 3. The thickness of the muscular stroma was also significantly less in Groups 3 and 4 than in Group 2 (Fig. 2). The folded tubules of Group 4 hamsters were more highly folded then were tubules in the other 3 groups.

Stereological characteristics of the ventral prostate were very similar in all 4 groups, with no consistent pattern being apparent.

\section{Discussion}

The purpose of this study was to examine whether mature dorsal and ventral prostate lobes that have undergone seasonal changes are different from those which do not. To this end, a stereological approach was used to compare the architecture of prostates allowed to regress and regrow (Group 3) with those which were not allowed to regress and regrow (Groups 2 and 4). As a baseline for comparisons, a group of young mature hamsters (12 weeks old, maintained in long-day photoperiods) in their first reproductive season (Group 1) was examined.

In the dorsal lobe of the prostate, no differences were seen between young hamsters (Group 1) and those in their second breeding season (Group 3). In other words, older dorsal prostates, which were allowed to regress and regrow, more closely resembled those of younger hamsters than did the dorsal prostates of older hamsters which were not subjected to these changes.

In the ventral prostate, there were even fewer differences between groups. This was not unexpected, as earlier work had demonstrated that this lobe does not change much, either in weight or in histology, with photoperiodic or endocrine manipulations (Ortiz, 1953; Schmidt \& Visek, 1977; Buzzell et al., 1985a, b).

In this study, there were a number of methodological changes from previous stereological studies of hamster prostates (Buzzell, 1985; Buzzell et al., 1985a). In the previous studies, only one type of secretory tubule was considered and its architecture was a reflection of the architecture both of distended and folded tubules. Recent studies have emphasized the fact that the distinctions 
between distended and folded tubules in the rodent prostate are real. Sugimura et al. (1986a, b) used whole mounts of mouse ventral and dorsolateral prostates to show that there is a clear demarcation between the main proximal ducts with little mucosal folding, and the distal secretory ducts with distinct and extensive mucosal folding. Whole mounts of hamster prostate glands reveal that a similar situation exists in the hamster dorsal (but not ventral) lobe (unpublished observations). In the dorsal lobe of the prostate, distended tubules empty into the urethra and, although secretory in nature (Toma \& Buzzell, 1988), their main role is to collect and store secretion produced in the folded tubules and to pass this secretion on to the urethra. These distinctions were not apparent in the ventral lobes of the hamster prostate (Toma \& Buzzell, 1988).

Distended tubules showed very few differences between groups. Sugimura et al. (1986b) also found that the distended proximal main ducts of mouse ventral and dorsolateral prostates are but little affected during castration-induced regression and regrowth following androgen administration. Folded tubules, which are more affected by castration and androgen administration (Sugimura et al., 1986b), showed more differences between groups in the present studies. The most obvious of these differences were significantly greater volume fractions, volumes, and thicknesses of the muscular stroma in prostates which had never regressed and regrown than in those from young hamsters or in prostates which had regressed and regrown (Figs $1 \& 2$ ).

The stroma of androgen-sensitive accessory sex glands, such as the prostate and the seminal vesicles, is very important, both as an inducer of growth and differentiation (Cunha et al., 1983), and as a target of sex steroids (Bruchovsky et al., 1981). In a seminal vesicle fibromuscular fraction from adult guinea-pigs, castration had no effect on DNA or collagen content. Exogenous dihydrotestosterone given to guinea-pigs increased fibromuscular collagen levels to supranormal values (Mariotti \& Mawhinney, 1982); oestradiol benzoate increased fibromuscular DNA and collagen to supranormal levels (Mariotti \& Mawhinney, 1981, 1982). Most studies do not distinguish between fibrous tissue (mainly in the interacinar tissue and lamina propria) and muscular tissue (in the muscular stroma of the hamster) and so it is difficult to draw direct comparisons between studies and across species. However, it appears that, in some rodents at least, stromal collagen content is controlled by both androgens and oestrogens and stromal cell numbers are controlled by oestrogens (Mariotti \& Mawhinney, 1981, 1982).

There do not appear to have been any previous studies of accessory sex glands subjected to short-day induced regression and redevelopment. However, several studies have examined prostatic regrowth induced by castration and androgen replacement. Few of these studies have looked in detail at different prostatic components. The studies by Mariotti \& Mawhinney $(1981,1982)$ have been mentioned. Huttunen et al. (1982) stereologically examined regrowth of the ventral prostate of the rat. They did not compare their data with those of controls which did not regress and regrow. They found large increases in the tubular parenchyma and, to a lesser extent, in the interacinar tissue. There was a large increase in the amount of epithelium and a greater increase in the total amount of lumen. This study did not consider the muscular stroma. DeKlerk \& Coffey (1978) determined that treatment of castrated rats for 10 days with testosterone restored epithelial cell size and stromal cell size and number but did not completely restore epithelial cell number in the ventral prostate.

In conclusion, the dorsal prostate of a seasonally breeding rodent, the Syrian hamster, regressed in short days but regrew spontaneously preparatory to the next breeding season. During this regrowth, the architecture of the gland was restored nearly to its normal state. The ventral prostate, in which seasonal regression is not marked, was also restored to its normal state. Glands prevented from regressing did not seem to have been adversely affected, although highly folded secretory tubules in the dorsal prostate had more muscular tissue in their walls.

This project was supported by the Alberta Cancer Board through the Alberta Heritage Savings Trust Fund, Applied Research-Cancer program (project H264). I thank Julie Haskins, Jean Toma and Gail O'Brien for assistance. 


\section{References}

Bruchovsky, N., McLoughlin, M.G., Rennie, P.S. \& To, M.P. (1981) Parital characterization of stromal and epithelial forms of $5 \alpha$-reductase in human prostate. In The Prostatic Cell: Structure and Function, Part A, pp. 161-175. Eds G. P. Murphy, A. A. Sandberg \& J. P. Karr. A. R. Liss, New York.

Buzzell, G.R. (1985) Light-microscopic stereology for the study of the ventral and dorsal prostates of the Syrian hamster: strategy, techniques, and applications. Am. J. Anat. 173, $299-308$.

Buzzell, G.R., Toma, J.G., O'Brien, M.G. \& Haskins, J. (1985a) Effects of short-day photoperiods and of castration on the structural integrity of the ventral and dorsal prostates of the Syrian hamster (Mesocricetus auratus). Am. J. Anat. 173, 309-319.

Buzzell, G.R., Toma, J.G., O'Brien, M.G. \& Haskins, J. (1985b) Responses of the accessory sex glands of the Syrian hamster to castration, short-day photoperiods and pinealectomy. In The Pineal Gland, Endocrine Aspects, pp. 115-120. Eds G. M. Brown \& S. D. Wainwright. Pergamon Press, Oxford.

Cunha, G.R., Fujii, H., Neubauer, B.L., Shannon, J.M., Sawyer, L. \& Reese, B.A. (1983) Epithelial-mesenchymal interactions in prostatic development. I. Morphological observations of prostatic induction by urogenital sinus mesenchyme in epithelium of the adult rodent urinary bladder. $J$. Cell Biol. 96, 16621670.

DeKlerk, D.P. \& Coffey, D.S. (1978) Quantitative determination of prostatic epithelial and stromal hyperplasia by a new technique. Biomorphometrics. Invest. Urol. 16, 241-245.

Goldman, B.D., Matt, K.S., Roychoudry, P. \& Stetson, M.H. (1981) Prolactin release in golden hamsters: photoperiod and gonadal influences. Biol. Reprod. 24, 287-292.

Hoffman, R.A. \& Reiter, R.J. (1965) Rapid pinealectomy in hamsters and other small rodents. Anat. Rec. 153, 19-22.

Huttunen, E., Romppanen, T. \& Helminen, H.J. (1982) Testosterone action on the ventral prostate lobe of the castrated rat as assessed with a stereologic morphometric method. Am. J. Anat. 165, 199-209.

Mariotti, A. \& Mawhinney, M. (1981) Hormonal control of accessory sex organ fibromuscular stroma. Prostate 2, 397-408.
Mariotti, A. \& Mawhinney, M. (1982) The hormonal maintenance and restoration of guinea pig seminal vesicle fibromuscular stroma. J. Urol. 128, 852-857.

Matt, K.S. \& Stetson, M.H. (1980) Comparison of serum hormone titers in golden hamsters during testicular growth induced by pinealectomy and photoperiodic stimulation. Biol. Reprod. 23, 893898.

Ortiz, E. (1953) The effects of castration on the reproductive system of the golden hamster. Anat. Rec. 117, 6591 .

Reiter, R.J. (1985) Pineal-reproductive interactions. In The Hamster. Reproduction and Behavior, pp. 99-118. Ed. H. I. Siegel. Plenum, New York.

Reiter, R.J. \& Hester, R.J. (1966) Interrelationships of the pineal gland, the superior cervical ganglia and the photoperiod in the regulation of the endocrine systems of hamsters. Endocrinology 79, 1168-1170.

Schmidt, T.J. \& Visek, W.J. (1977) The effects of castration on the ventral prostate of the outbred Syrian hamster. Proc. Soc. exp. Biol. Med. 155, 105-110.

Steger, R.W., Bartke, A. \& Goldman, B.D. (1982) Alterations in neuroendocrine function during photoperiod induced testicular atrophy and recrudescence in the golden hamster. Biol. Reprod. 26, 437-444.

Sugimura, Y., Cunha, G.R. \& Donjacour, A.A. (1986a) Morphogenesis of ductal networks in the mouse prostate. Biol. Reprod. 34, 961-971.

Sugimura, Y., Cunha, G.R. \& Donjacour, A.A. (1986b) Morphological and histological study of castrationinduced degeneration and androgen-induced regeneration in the mouse prostate. Biol. Reprod. 34, 973-983.

Toma, J.G. \& Buzzell, G.R. (1988) Fine structure of the ventral and dorsal lobes of the prostate in the young adult Syrian hamster. Am. J. Anat. 181, 132-140.

Turek, F.W., Elliott, J.A., Alvis, J.D. \& Menaker, M. (1975) Effect of prolonged exposure to non-stimulatory photoperiods on the activity of the neuroendocrine-testicular axis of golden hamsters. Biol. Reprod. 13, 475-481.

Weibel, E.R. (1979) Stereological Methods. Volume 1. Practical Methods for Biological Morphometry. Academic Press, London. 